\title{
Invariância fatorial, sensibilidade e diferenças da medida de ansiedade, estresse e depressão (DASS-21) em trabalhadores brasileiros
}

Factorial invariance, sensitivity and differences of the measure of Anxiety, stress and depression in Brazilian workers

Invarianza factorial, sensibilidad y diferencias de la medida de ansiedad, estrés y depresión en trabajadores brasileños

Recebido: 28/04/2021 | Revisado: 06/05/2021 | Aceito: 07/06/2021 | Publicado: 21/06/2021

Nilton S. Formiga

ORCID: https://orcid.org/0000-0003-4907-9736 Universidade Potiguar, Brasil

E-mail: nsformiga@yahoo.com

Juliana Bianca Maia Franco

ORCID: https://orcid.org/0000-0002-7566-2688 Universidade Potiguar, Brasil

E-mail: juliana_franco_@hotmail.com

Heitor César Costa Oliveira

ORCID: https://orcid.org/ 0000-0003-4778-6613 Universidade Potiguar/

Laureate International Universities, Brasil

E-mail: cartoriosbrasil@gmail.com

Gleyssielle Lira Prochazka

ORCID: https://orcid.org/0000-0001-6083-1306 Universidade Potiguar, Brasil

E-mail: gleyssielle.lira@gmail.com

Thalita Kelle Pires Beserra

https://orcid.org/0000-0003-2693-8702

Universidade Potiguar, Brasil

E-mail: tpiresb@gmail.com

Cristienne Gonçalves Pereira Valin

ORCID: https://orcid.org/0000-0002-7928-4856

Universidade Potiguar, Brasil

E-mail: cris2n@hotmail.com

Sara Ruth Araújo Grangeiro

ORCID: https://orcid.org/0000-0001-7446-6020 Universidade Potiguar, Brasil

E-mail: sararuthmbc@hotmail.com

Rodrigo L. Nascimento

ORCID: https://orcid.org/0000-0002-1291-9992

Universidade Potiguar, Brasil

E-mail: rodrigonascimento070187@gmail.com

\begin{abstract}
Resumo
Ao longo dos tempos as relações entre as dimensões afetivas negativas, ansiedade e depressão, têm sido consideradas importantes tanto do ponto de vista teórico como no da saúde mental e, por isso, constituem dimensões clássicas na psicologia e na psicopatologia. A operacionalização dos modelos existentes levou à construção de medidas tais como a escala aqui em estudo, a Depression Anxiety Stress Scale (DASS) que avalia sintomas de depressão, ansiedade e estresse em 21 itens, assinalando em uma escala de frequência de quatro pontos, em que medida experimentaram cada sintoma na última semana. Trata-se de um estudo descritivo, exploratório e correlacional, de abordagem quantitativa envolvendo trabalhadores nas cidades de e João Pessoa-PB e Natal-RN. Considerando uma coleta de dados do tipo intencional, através do método bola de neve, participaram da amostra 219 trabalhadores, com a maioria: do setor privado (47\%), da cidade de João Pessoa-PB (55\%), casados (44\%), 66\% mulheres, com especialização (36\%) e idades acima de 21 anos. Estes responderam os dados sociodemográficos e a escala DASS-21. Os resultados revelaram, que o modelo trifatorial oblíquo ajustado, quando comparados aos demais modelos estabelecidos, apresentou melhores indicadores psicométricos. Bem como, sensibilidade diagnóstica para os trabalhadores. A escala em questão é adequada, confiável e invariável, garantindo a capacidade avaliativa deste construto.
\end{abstract}

Palavras-chave: DASS-21; Transtorno emocional; Trabalhadores; Análise confirmatória. 


\begin{abstract}
Over time the relations between negative affective dimensions, anxiety and depression have been considered important from both theoretical and mental health points of view and, therefore, constitute classic dimensions in psychology and psychopathology. The operationalization of the existing models led to the construction of measures such as the scale focus of this study, the Depression Anxiety Stress Scale (DASS) which evaluates symptoms of depression, anxiety and stress in 21 items, indicating on a four-point frequency scale how much one experienced each symptom the previous week. This is a descriptive, exploratory and correlational study with a quantitative approach involving workers in the cities of and João Pessoa-PB and Natal-RN. Considering a collection of intentional data, using the snowball method, 219 workers participated in the sample, with the majority: from the private sector (47\%), from the city of João Pessoa-PB (55\%), married (44\%), 66\% women, with Specialization $(36 \%)$ and over the age of 21 years old. These workers answered the sociodemographic data and the DASS-21 scale. The results revealed that the adjusted oblique trifactor model, when compared to the other established models, presented better psychometric indicators. As well as diagnostic sensitivity for workers. The scale in question is adequate, reliable and invariable, ensuring the evaluative capacity of the construct.
\end{abstract}

Keywords: DASS-21; Emotional disorder; Workers; Confirmatory analysis.

\begin{abstract}
Resumen
A lo largo del tiempo, las relaciones entre las dimensiones afectivas negativas, la ansiedad y la depresión se han considerado importantes tanto desde el punto de vista teórico como mental y, por lo tanto, constituyen dimensiones clásicas en psicología y psicopatología. El funcionamiento de los modelos existentes condujo a la construcción de medidas como la escala en estudio, la Escala de Estrés de Ansiedad por Depresión (DASS) que evalúa los síntomas de depresión, ansiedad y estrés en 21 ítems, indicando en una escala de frecuencia de cuatro puntos en cuánto experimentó cada síntoma en la última semana. El estudio es de carácter descriptivo, exploratorio y correlacional con un enfoque cuantitativo que involucra a trabajadores en las ciudades de João Pessoa-PB y Natal-RN. Considerando una recogida de datos intencionales, utilizando el método de bola de nieve, 219 trabajadores participaron de la muestra, con la mayoría: del sector privado (47\%), de la ciudad de João Pessoa-PB (55\%), casados (44\%), 66\% mujeres, con especialización (36\%) y mayores de 21 años. Estos respondieron los datos sociodemográficos y la escala DASS-21. Los resultados revelaron que el modelo trifactorial oblicuo ajustado, en comparación con los otros modelos establecidos, presentaba mejores indicadores psicométricos. Así como la sensibilidad diagnóstica para trabajadores. La escala en cuestión es adecuada, confiable e invariable, asegurando la capacidad evaluativa del constructo.
\end{abstract}

Palabras clave: DASS-21; Trastorno emocional; Trabajadores; Análisis confirmatorio.

\title{
1. Introdução
}

No cenário internacional, com base na produção científica ou na mídia em geral, é possível acompanhar o quanto o tema do transtorno emocional na população em geral ou em grupos tão específicos (por exemplo, crianças, idosos, pessoas em vulnerabilidade e profissionais dos mais diversos) tem sido uma preocupação para pesquisas que destaquem tanto explicações teóricas quanto aplicabilidade de seus conceitos para o mundo do trabalho (cf. Hahn, Hantikainen, Needham, Kok, Dassen, \& Halfens, 2012; Borim, 2013; Edward, Ousey, Warelow, \& Lui, 2014; Spector, Zhou, \& Che, 2014; Morin, 2016).

Entretanto, no Brasil, este fenômeno psicológico em trabalhadores nas mais variadas profissões, ainda tem permitido o desenvolvimento e verificação de medidas sobre o transtorno emocional com maiores detalhes de indicadores psicométricos para uma avaliação mais consistentes e confiável relativo a uma diagnose (Vasconcellos, Abreu, \& Maia, 2012; Vasconcellos, Griep, Lisboa, \& Rotenberg, 2012).

Essa condição tem sido urgente, justamente, por esse fenômeno apresentar uma certa 'invisibilidade' quanto as ocorrências, consequências emocionais e comportamentais preditivas, os quais, bastante variados, tendo em sua maioria, justificativas causais quanto elementos 'quase naturais' existentes nos ambientes laborais e na vida das pessoas, subestimando a magnitude e intensidade do problema (cf. Leite \& Souza, 2006; Brixner, Formiga, \& Navarro, 2019).

As consequências negativas do transtorno emocional na saúde dos trabalhadores e, sobre a assistência prestada, têm sido evidenciadas através de sintomas de estresse, baixa autoestima e desmotivação das vítimas (Edward, Ousey, Warelow, \& Lui, 2014; Batista, Campos, Reis, \& Schall, 2011). Além disso, os transtornos psíquicos menores, também, têm sido associados às vítimas de violência no trabalho, os quais são descritos por sintomas psiquiátricos não psicóticos, como 
ansiedade, insônia, tristeza, fadiga, esquecimento, obstáculos de concentração, irritabilidade, queixas somáticas e até assédio moral (Vasconcellos, Griep, Lisboa, \& Rotenberg, 2012; Pai, Lautert, Souza, Marziale, \& Tavares, 2015; Formiga, Lima, Franco, \& Pereira, 2020).

De forma geral, as relações entre as dimensões afetivas de caráter negativos, relativos à ansiedade e depressão, têm sido consideradas importantes enquanto teórico quanto de aplicabilidade na bem-estar mental e por tal condição, constituem dimensões clássicas na Psicologia e na psicopatologia no procedimento de avaliação e intervenção, pois, considera-se que ansiedade e depressão tem uma relação bastante estreita entre eles. Até os anos de 1900 as perturbações de ansiedade não se separaram de outras perturbações do humor, com os menores casos denominados de neurastenia (Gelder, Gath, Mayou, \& Cowen, 1996; Gazalle, Lima, Tavares, \& Hallal, 2004; McCauley, Katon, Russo, Richardson, \& Lozano, 2007; Melo, Siebra, \& Moreira, 2017).

Apesar destes construtos serem considerados independentes, ainda é reconhecido a existência de uma sobreposição entre eles, seja na visão da saúde mental ou de avaliação de construto (diagnóstico ou epidemiológico) responsáveis por uma variedade de perturbações designadas no Manual de Diagnóstico e Estatístico - DSM V, as quais, associam-se ao mesmo medicamento ou tratamento psicossocial tornando complexo a diferenciação empiricamente do diagnóstico (cf. Watson, Clark, Weber, Assenheimer, Staruss, \& McCormick, 1995; Watson, Weber, Assenheimer, Clark, Staruss, \& McCormick, 1995; Brown, Chorpita, \& Barlow, 1998; Baptista \& Carneiro, 2011; American Psychiatric Association, 2014; Hamaideh, 2017).

Diante dessas ambivalências avaliativas, na verificação desses transtornos afetivos, um estudo desenvolvido por estudo Clark e Watson (1991; Terra, 2010; cf. Martins, Silva, Maroco, \& Campos, 2019), propôs um modelo tripartido em que os sinais de ansiedade e depressão se alinham em arcabouços basais: uma primitiva estrutura designada por distress ou afeto negativo, inclui sintomas relativamente inespecíficos, experimentados tanto por indivíduos deprimidos quanto ansiosos, incluído ainda humor deprimido e ansioso, assim como, insônia, desconforto ou insatisfação, irritabilidade e dificuldade de concentração.

Estes sintomas inespecíficos são responsáveis pela forte associação entre as medidas de ansiedade e depressão. Para além deste fator inespecífico, a ansiedade e a depressão constituiriam as outras duas estruturas, com a crise somática e a hiperatividade como específicas da ansiedade, e a anedonia e a lacuna de afeição positiva como relativamente específicas da depressão. A operacionalização do modelo tripartido levou à construção de medidas tais como a escala avaliada no presente artigo, a Depression Anxiety Stress Scale (DASS) (Pais-Ribeiro, Honrado, \& Leal, 2004).

A escala de depressão, ansiedade e estresse [Depression, Anxiety and Stress Scale, Short Form (DASS-21)] foi criada por Lovibond e Lovibond (1995) com o alvo de aferir e distinguir, ao extremo, os sinais de ansiedade e depressão, bem como, estabelecer a dimensão da avaliação do estresse como complemento da medida de transtorno afetivo. Segundo Silva, Passos, Oliveira, Palmeira Pitangui e Araújo (2016) a versão reduzida da Escala de Ansiedade Depressão e Estresse-21, proporcionou uma medida de autorrelato de sinais de ansiedade, depressão e estresse. Baseada no padrão tripartido em que os sinais de ansiedade e depressão se ajuntam em três arcabouços básicos: presença de afeição negativa, como humor deprimido, insônia, desconforto e irritabilidade, incluídos na depressão e a ansiedade; sintomas de anedonia, ausência de afeto positivo, específicos na depressão e tensão somática e hiperatividade, relativo à ansiedade (Watson et al., 1995).

Foi Machado e Bandeira (2013), quem adaptaram para o contexto brasileiro a escala DASS21; esta, além de adaptada, foi validade em uma amostra de adultos em diversas regiões do país. Revelou alfas que variaram de 0,92 a 0,96 para os três fatores. Ainda com uma amostra de brasileiros, porém, considerando sujeitos clinicamente diagnosticados, Vignola e Tucci (2014) observaram evidências de validade com alfas que estiveram de 0,86 a 0,92.

Nesse mesmo cenário a pesquisa de Martins, Silva, Maroco e Campos (2019) verificaram com 1.042 estudantes, com idades entre 18 e 35 anos, homens e mulheres, identificaram também, que a DASS-21 foi adequada, contudo o item 2 
apresentou baixo peso fatorial, sendo, portanto, excluído. Em relação ao grau de acometimento dos estudantes pela depressão, ansiedade e estresse, verificou-se que 4,7\%, 1,7\% e 4,5\% dos estudantes apresentaram, respectivamente, escores moderados a extremamente severos. Não houve diferença significativa entre as prevalências dos estados emocionais avaliados segundo o sexo.

A partir dos estudos, supracitados, o objetivo principal desse artigo trata-se de verificar tanto a qualidade psicométrica da escala DASS - 21, sua invariância fatorial e sensibilidade diagnóstica em uma amostra de trabalhadores. Com base nas considerações teóricas expostas acima, espera-se que a estrutura fatorial com três fatores, previamente proposta por Lovibond e Lovibond (1995), ao compará-las as outras estruturas fatoriais, apresente melhores indicadores psicométricos.

\section{Metodologia}

\section{Amostra}

Trata-se de um estudo descritivo, exploratório e correlacional, de abordagem quantitativa envolvendo trabalhadores das mais distintas categorias profissionais; para isso utilizou-se à abordagem não probabilística em bola de neve, fazendo-se necessário avaliar o 'n' amostral mais adequado para a realização do estudo. Sendo assim, utilizou-se o pacote estatístico GPower 3.1 para verificar a qualidade da amostra no estudo (Faul, Erdfelder, Lang, \& Buchner, 2007).

A amostra final de 463 trabalhadores brasileiros, na cidade de João Pessoa-PB (43\%) e de Natal-RN (57\%), revelou-se ser suficiente para a referida pesquisa, tendo como indicadores da qualidade amostral: $t \geq 1,98 ; \pi=0,93 ; \mathrm{p}<0,05$.

Participaram do estudo 463 trabalhadores, distribuídos em funcionários do setor público e privado, do sexo masculino (42\%) e do sexo feminino (58\%), de 21 a 59 anos, com $42 \%$ deles tendo uma renda econômica acima de 4.000,00 reais. Para a permanência na amostra, considerou-se a pessoa que, quando consultada, in loco ou por meio de questionários eletrônicos inseridos na plataforma de acesso google forms, os quais, eram enviados por e-mail e compartilhado em redes sociais.

\section{Instrumentos}

Os sujeitos responderam aos seguintes questionários:

Escala de Ansiedade, depressão e estresse (em inglês, DASS-21): trata-se de um instrumento que foi desenvolvido Lovibond e Lovibond (1995), constituído por um conjunto de três sub-escalas, respondida na escala tipo Likert, de 4 pontos, variando de $0=$ não se aplica totalmente a 4 = aplica-se totalmente. Cada sub-escala é composta por 7 itens, destinados a avaliar os estados emocionais de depressão, ansiedade e estresse.

A DASS foi desenvolvida com base no modelo tripartido, o qual, se organiza e estrutura em conceito e medida nos sintomas de cada transtorno destacado aqui; a saber: uma das dimensões, por exemplo, a depressão avalia a presença de sintomas que salientam afeto negativo, como inércia, falta de prazer e interesse/envolvimento, disforia, falta de autodepreciação, desvalorização da vida e desânimo. A ansiedade, excitação do sistema nervoso autónomo; efeitos musculoesqueléticos; ansiedade situacional; experiências subjetivas de ansiedade. Finalmente, a subescala de estresse avalia dificuldade em relaxar; excitação nervosa; fácil perturbação/agitação; irritabilidade/reação exagerada e impaciência. Esta medida tinha como objetivo para autores que a desenvolveram (cf. Lovibond \& Lovibond, 1995) mensurar e diferenciar, ao máximo, os sintomas de ansiedade e depressão em sujeitos não clínicos.

A sua construção tomou-se como referência o modelo tripartido; este propõem uma estrutura fatorial com base no conceito e medida dos sintomas de ansiedade e depressão (cf. Patias, Machado, Bandeira, \& Dell'Aglio, 2016); sendo assim, é possível encontrar itens que com características da presença de afeto negativo (a saber: humor deprimido, insônia, desconforto e irritabilidade), destacando sintomas inespecíficos incluídos tanto na depressão quanto na ansiedade; e outros, que constituem 
estruturas que representam sintomas específicos para depressão (anedonia, ausência de afeto positivo); outros mais, referem-se aos sintomas específicos de ansiedade (tensão somática e hiperatividade) (Watson et al., 1995).

Na DASS-21, os respondentes indicam o grau com que vivenciaram cada um dos sintomas descritos nos itens, para tanto, estes, devem fazer referência o quanto sentiram isso durante a última semana (isto é, a semana anterior) e indicar na escala do tipo Likert de 4 pontos, variando da seguinte forma: de 0 (não se aplica a mim) e 3 (aplica-se muito a mim, ou a maior parte do tempo).

Quanto a formação dos fatores, estes, são computados a partir da soma dos itens pertencentes a cada um deles; no que se refere a pontuação total do construto transtorno emocional, soma-se todos os 21 itens. A estrutura fatorial da DASS-21, não somente é confiável, mas, também, vem se mantendo quanto a sua fidedignidade e validade convergente e discriminante (cf. Patias, Machado, Bandeira, \& Dell'Aglio, 2016).

Além desse instrumento, um pequeno questionário foi também inserido para verificar alguns dados sociodemográficos como idade, sexo, renda econômica etc.

\section{Procedimentos e administração do instrumento}

Colaboradores com experiência prévia na administração do DASS-21 foram responsabilizados pela coleta dos dados, e apresentaram-se in loco ou por meio de questionários eletrônicos inseridos na plataforma de acesso google forms, os quais, eram enviados por e-mail e compartilhado em redes sociais. Para isso, informava-lhes que o instrumento se referia ao conhecimento das opiniões e os comportamentos sobre as situações descritas relativa aos trabalhadores em distintas empresas.

Solicitou-se a colaboração voluntária das pessoas no sentido de responderem um breve questionário. Após ficarem cientes das condições de participação na pesquisa, assinaram um termo de Consentimento Livre e Esclarecido. Foi-lhes dito que não havia resposta certa ou errada. A todos foi assegurado o anonimato das suas respostas informando que estas seriam tratadas em seu conjunto. A DASS-21 foi respondida individualmente quando in loco em ambas as cidades, bem como, na forma de compartilhamento eletrônico nas redes sociais e de mensagens (Facebook, Instagram e WhatsApp), ou ainda via email; nestes, o instrumento foi hospedado e disponibilizado via doc.google.com./forms.

Apesar de o instrumento ser auto-aplicável, contando com as instruções necessárias para que possam ser respondidos, os colaboradores na aplicação estiveram presentes durante toda à administração do instrumento, quando em local físico, e por e-mail ou rede social, quando no meio eletrônico, para retirar eventuais dúvidas ou realizar esclarecimentos que se fizessem indispensáveis. Um tempo médio de 20 minutos foi suficiente para concluir essa atividade.

Todos os procedimentos adotados nesta pesquisa seguiram as orientações previstas na Resolução 466/2012 do CNS e na Resolução 016/2000 do Conselho Federal de Psicologia para as pesquisas com seres humanos (Conselho Nacional de Saúde [CNS], 2012; ANPEPP, 2000), aprovado pelo Comitê de Ética em Pesquisa da Plataforma Brasil, sob Certificado de Apresentação para Apreciação Ética CAAE 15827919.0.0005296.

\section{Análise dos dados}

Quanto à análise dos dados, realizou-se, além das análises descritivas, efetuou-se também, uma análise fatorial confirmatória afim de avaliar a estrutura fatorial do modelo em relação a DASS-21. Considerou-se como entrada a matriz de covariâncias o estimador ML (Maximum Likelihood). Sendo um tipo de análise estatística mais criteriosa e rigorosa, buscou-se avaliar a estrutura teórica do DASS-21 com três fatores. A seguir serão apresentados esses indicadores:

- O $\chi^{2}$ (qui-quadrado) testa a probabilidade do modelo teórico se ajustar aos dados; Raiz Quadrada Média Residual (RMR), que indica o ajustamento do modelo teórico aos dados, na medida em que a diferença entre os dois se aproxima de zero; O Goodness-of-Fit Index (GFI) e o Adjusted Goodness-of-Fit Index (AGFI) são análogos ao $R^{2}$ na regressão múltipla e, 
portanto, indicam a proporção de variância-covariância nos dados explicada pelo modelo; A Root-Mean-Square Error of Approximation (RMSEA), com seu intervalo de confiança de 90\% (IC90\%), é considerado um indicador de "maldade" de ajuste, isto é, valores altos indicam um modelo não ajustado; O Comparative Fit Index (CFI) - compara de forma geral o modelo estimado ao modelo nulo, considerando valores mais próximos de um como indicadores de ajustamento satisfatório; o Expected Cross-Validation Index (ECVI) e o Consistent Akaike Information Criterion (CAIC) são indicadores geralmente empregados para avaliar a adequação de um modelo determinado em relação a outro. Valores baixos do ECVI e CAIC expressam o modelo com melhor ajuste (Byrne, 1989; Joreskög \& Sörbom, 1989; Hair, Anderson, Tatham \& Black, 2005).

Realizaram-se, tanto o cálculo de confiabilidade composta (CC) quanto da variância média extraída (VME); no primeiro indicador exige-se que o nível do escore seja acima de 0,70, enquanto no segundo indicador é preciso um nível acima de 0,50 (Hair, Anderson, Tatham \& Black, 2005); realizou-se também, análise de Curva ROC, com objetivo de avaliar as especificidades e sensibilidade diagnóstica do instrumento (Oliveira \& Andrade, 2002; Margotto, 2002).

\section{Resultados}

Inicialmente, após a inspeção visual dos valores máximos e mínimos das escalas as condições dos critérios mínimos que atendesse aos pressupostos dos cálculos a serem realizados e devido a diversidade amostral dos respondentes quanto a variação de profissões participantes da pesquisa, realizou-se também, algumas análises para avaliar a qualidade da amostra coletada:

- quanto aos dados omissos do estudo, eles não foram acima de 5\%, definido por Tabachnick e Fidell (2001), podendo com isso, substituir pela média ou moda dos dados da amostra quando estes for missing ou ter erro de digitação;

- a multicolinearidade entre as variáveis, estiveram dentro dos parâmetros definidos por Tabachnick e Fidell (2001) [r $\leq 0,90$,$] ; neste estudo variou de 0,05$ a 0,86 . Quanto aos outliers multivariados na amostra, o teste de normalidade de Kolmogorov-Smirnov (K-S), destinado a análise de amostras iguais ou inferiores a 100 sujeitos, apresentou uma normalidade ( $\mathrm{S}-\mathrm{W}=0,83$ ) da amostra a um $\mathrm{p}<0,14$ (cf. Nascimento, Tibana, Melo, \& Prestes, 2015).

- por fim, assumindo uma avaliação adicional, testou-se a tendência de não resposta, usando o procedimento de Armstrong e Overton (1977), através do qual se avaliou as diferenças entre os primeiros (n1 = 10) e os últimos (n2 = 10) participantes da pesquisa e que, com base no teste de Levene verificou a igualdade das variâncias e com o teste $t$ a igualdade dos meios, observou-se a não existência de resultados significativos entre os escores médios dos constructos. Confirmado a qualidade das medidas e dos construtos em questão, procurou-se seguir para avaliação do objetivo principal da pesquisa, o qual, refere-se à verificação da estrutura fatorial da DASS-21 em uma amostra com trabalhadores em distintas profissões.

Verificou-se a estrutura fatorial considerando os achados de Lovibond e Lovibond (1995), Machado e Bandeira (2013) e Patias, Machado, Bandeira e Dell'Aglio (2016), no que se refere a organização fatorial da DASS-21; para garantir a qualidade da medida gerou-se tanto o modelo proposto (estrutural trifatorial oblíqua ajustada) quanto modelos comparativos (isto é, unifatorial, bifatorial [pois eram proposições de alguns estudos, os quais, sugerindo a unifatorialidade da dimensão stress e ansiedade], ortogonal [neste proposta, consideraram-se os fatores não relacionados] e o oblíquo não ajustado). Os resultados revelaram que o modelo trifatorial oblíquo ajustado, quando comparados aos demais modelos estabelecidos, apresentou melhores indicadores psicométricos, destacado em negrito (ver Tabela 1). 
Research, Society and Development, v. 10, n. 7, e26910715572, 2021

(CC BY 4.0) | ISSN 2525-3409 | DOI: http://dx.doi.org/10.33448/rsd-v10i7.15572

Tabela 1: Indicadores psicométricos da comparação da estrutura fatorial do DASS-21.

\begin{tabular}{|c|c|c|c|c|c|c|c|c|c|}
\hline \multirow[t]{2}{*}{ Modelos } & \multicolumn{4}{|c|}{ Medidas de ajuste absoluto } & \multicolumn{3}{|c|}{$\begin{array}{l}\text { Medidas de ajuste } \\
\text { incremental }\end{array}$} & \multicolumn{2}{|c|}{$\begin{array}{l}\text { Medidas de ajuste } \\
\text { parcimonioso }\end{array}$} \\
\hline & $\chi^{2} / \mathrm{gl}$ & RMR & GFI & AGFI & CFI & TLI & $\begin{array}{l}\text { RMSEA } \\
\text { (intervalo) }\end{array}$ & CAIC & $\begin{array}{c}\text { ECVI } \\
\text { (intervalo) }\end{array}$ \\
\hline Unifatorial & 8,96 & 0,10 & 0,55 & 0,63 & 0,88 & 0,87 & $\begin{array}{c}0,13 \\
(0,12-0,14)\end{array}$ & 1992,40 & $\begin{array}{c}3,84 \\
(3,57-4,14)\end{array}$ \\
\hline Bifatorial & 7,59 & 0,54 & 0,83 & 0,74 & 0,91 & 0,89 & $\begin{array}{c}0,12 \\
(0,11-0,13)\end{array}$ & 1727,34 & $\begin{array}{c}3,11 \\
(2,68-3,36)\end{array}$ \\
\hline $\begin{array}{l}\text { Trifatorial } \\
\text { Ortogonal }\end{array}$ & 11,07 & 1,27 & 0,76 & 0,70 & 0,85 & 0,84 & $\begin{array}{c}0,15 \\
(0,14-0,16)\end{array}$ & 2392,60 & $\begin{array}{c}4,71 \\
(4,40-5,04) \\
\end{array}$ \\
\hline $\begin{array}{l}\text { Trifatorial } \\
\text { Oblíquo não } \\
\text { ajustado }\end{array}$ & 3,34 & 0,05 & 0,88 & 0,85 & 0,97 & 0,96 & $\begin{array}{c}0,07 \\
(0,06-0,08)\end{array}$ & 942,02 & $\begin{array}{c}1,54 \\
(1,38-1,71)\end{array}$ \\
\hline $\begin{array}{l}\text { Trifatorial } \\
\text { Oblíquo } \\
\text { ajustado }\end{array}$ & 1,92 & 0,04 & 0,94 & 0,92 & 0,99 & 0,99 & $\begin{array}{c}0,04 \\
(0,04-0,05)\end{array}$ & 766,59 & $\begin{array}{c}0,97 \\
(0,87-1,09)\end{array}$ \\
\hline
\end{tabular}

Fonte: Autores.

A fim de gerar propor uma avaliação parcimoniosa do modelo fatorial pretendido, verificaram-se os indicadores AIC, BIC e BCC, estes acompanham o CAIC e ECVI, os quais, destinados a verificação da adequabilidade do pretenso modelo, especialmente, quando se trata da comparação entre estruturas fatoriais (cf. Marôco, 2010). Como observado na Tabela 2, os melhores indicadores estiveram para o modelo trifatorial obliquo ajustado, destacado em negrito.

Tabela 2: Indicadores psicométricos de parcimônia para a comparação da estrutura fatorial da DASS-21.

\begin{tabular}{cccc}
\hline Modelos & \multicolumn{3}{c}{ Indicadores de Parcimônia } \\
\cline { 2 - 4 } & AIC & BIC & BCC \\
\hline Unifatorial & 1776,62 & 1950,40 & 1780,81 \\
\hline Bifatorial & 1434,49 & 1670,64 & 1440,19 \\
\hline $\begin{array}{c}\text { Trifatorial Ortogonal } \\
\text { Trifatorial Oblíquo } \\
\text { não ajustado }\end{array}$ & 2176,82 & 2350,60 & 2181,02 \\
\hline Trifatorial Oblíquo ajustado & 710,82 & 897,02 & 715,32 \\
\hline
\end{tabular}

Fonte: Autores.

Na Figura 1, é possível observar que as saturações (Lambdas, $\lambda$ ) estão no intervalo 0 - 1, destacado a não existência de problemas de estimação para o modelo; além de estarem inseridas nestes parâmetros, elas são estatisticamente diferentes de zero $(t>1,96, p<0,05)$, garantindo a qualidade da validade da estrutura fatorial do DASS-21.

Ainda neste contexto de validade, destaca-se, a confiabilidade composta (CC), tendo como critério escore acima de 0,70 e a variância média extraída (VME) que deverá apresentar um escore acima de 0,50. Observou-se que o CC e o VME estiveram acima do exigido na literatura estatística (Hair et. al., 2005; Marôco, 2010; Lattin et. al., 2011) variando de 0,56 a 0,91, tanto para a dimensão total do DASS-21, quanto a especificidades das dimensões desta (ansiedade, depressão e estresse) condição, a qual, evidencia, respectivamente, a confiabilidade e validade convergente do construto avaliado. 
Figura 1: Representação gráfica da estrutura fatorial do DASS-21.

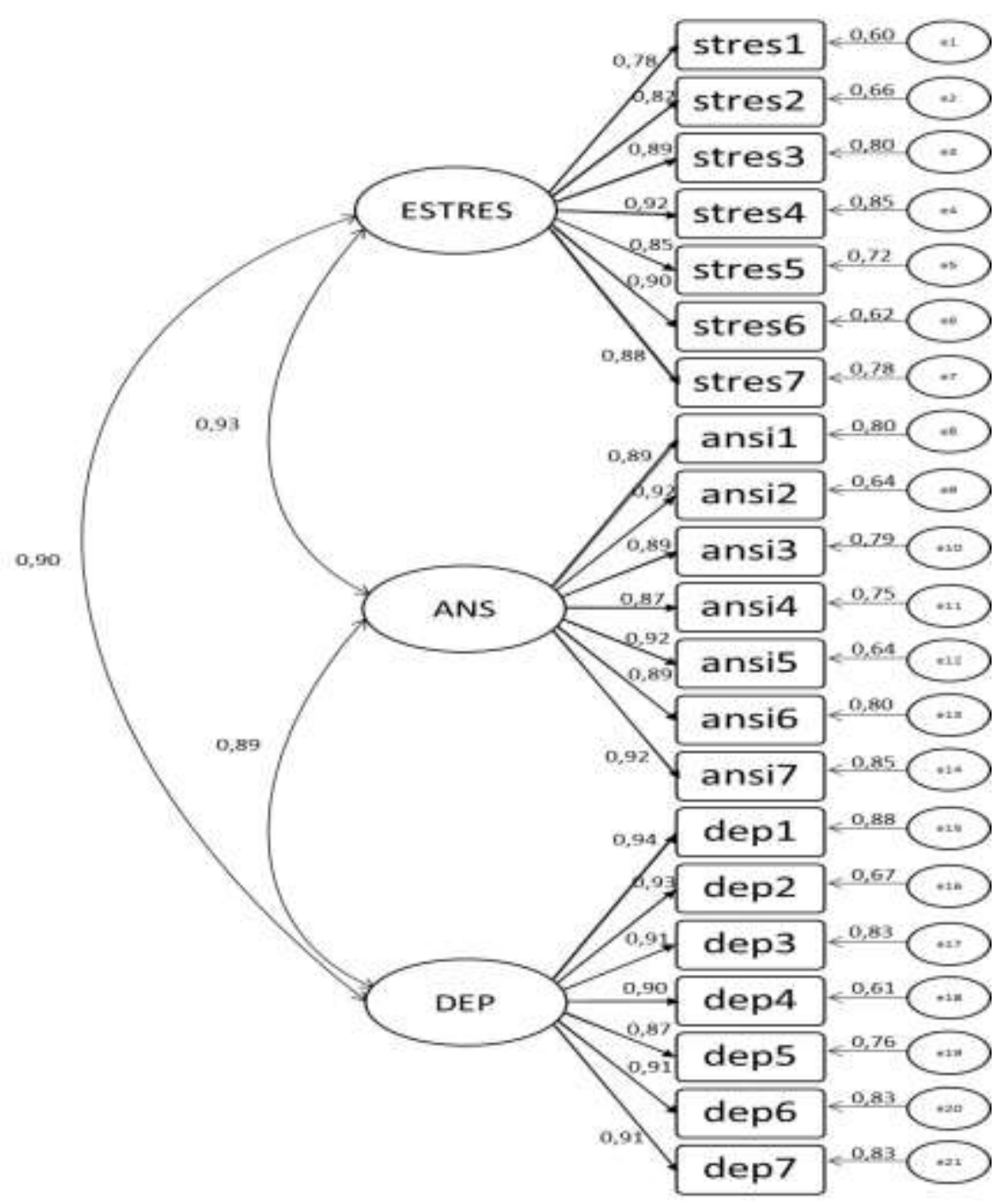

Fonte: Autores.

Os resultados, referentes a estrutura com três fatores, foram confirmados ao observar as estimativas preditivas, a partir da análise de regressão; sendo assim, o modelo proposto, a partir da identificação das variáveis da DASS-21, apresentou uma razão critério que, não apenas correspondia ao se esperava estatisticamente, mas, que foi diferente de zero $(t>1,96, p<0,05)$, com todas elas significativas (ver tabela 3 )

Na mesma Tabela 3, a partir do indicador VIF (definida em português, Inflação da Variância do Fator - IVF), com base no fator de tolerância entre as variáveis, o qual deverá apresentar escores que não excedam um valor acima de 5 (Hair, Tatham, Anderson, \& Black, 2005; Marôco, 2010), observou-se que, para cada item, o VIF encontram-se no limite de estimação exigido e significativos $(\mathrm{p}<0,01)$ não existência multicolinearidade da estimação do modelo fatorial pretendido. 
Tabela 3: Indicadores das estimativas preditivas da associação itens-fator da DASS-21.

\begin{tabular}{ccc|ccccc}
\hline Variáveis & $<---$ & \multirow{2}{*}{ fatores } & Estimativa & d.p. & $\begin{array}{c}\text { Razão } \\
\text { Critério }\end{array}$ & p-valor & VIF \\
\hline stres1 & $<---$ & ESTRES & 1,000 & --- & --- & --- & 2,63 \\
stres2 & $<---$ & ESTRES & 1,027 & 0,052 & 19,624 & 0,001 & 3,05 \\
stres3 & $<---$ & ESTRES & 1,207 & 0,055 & 21,805 & 0,001 & 3,71 \\
stres4 & $<---$ & ESTRES & 1,308 & 0,058 & 22,567 & 0,001 & 3,33 \\
stres5 & $<---$ & ESTRES & 1,057 & 0,053 & 19,921 & 0,001 & 2,18 \\
stres6 & $<---$ & ESTRES & 1,233 & 0,055 & 22,360 & 0,001 & 2,60 \\
stres7 & $<---$ & ESTRES & 1,050 & 0,045 & 23,458 & 0,001 & 3,04 \\
ansi1 & $<---$ & ANS & 1,000 & --- & --- & --- & 3,01 \\
ansi2 & $<---$ & ANS & 1,109 & 0,036 & 30,892 & 0,001 & 3,95 \\
ansi3 & $<---$ & ANS & 1,046 & 0,037 & 28,038 & 0,001 & 3,33 \\
ansi4 & $<---$ & ANS & 0,961 & 0,037 & 26,243 & 0,001 & 3,61 \\
ansi5 & $<---$ & ANS & 1,021 & 0,034 & 30,358 & 0,001 & 3,82 \\
ansi6 & $<---$ & ANS & 1,067 & 0,036 & 29,440 & 0,001 & 3,22 \\
ansi7 & $<---$ & ANS & 1,123 & 0,035 & 31,946 & 0,001 & 2,43 \\
dep1 & $<---$ & DEP & 1,000 & --- & --- & --- & 3,77 \\
dep2 & $<---$ & DEP & 1,004 & 0,023 & 43,165 & 0,001 & 3,84 \\
dep3 & $<---$ & DEP & 0,964 & 0,026 & 37,771 & 0,001 & 2,79 \\
dep4 & $<---$ & DEP & 0,983 & 0,030 & 32,594 & 0,001 & 3,44 \\
dep5 & $<---$ & DEP &, 956 & 0,034 & 28,362 & 0,001 & 2,42 \\
dep6 & $<---$ & DEP & 1,057 & 0,030 & 35,234 & 0,001 & 3,02 \\
dep7 & $<---$ & DEP & 0,935 & 0,028 & 33,982 & 0,001 & 3,45 \\
\hline
\end{tabular}

Fonte: Autores.

Considerando esses resultados, avaliou-se a invariância da estrutura trifatorial; com base nos parâmetros dos itens em função da estrutura tetrafatorial oblíqua ajustada da mensuração do DASS-21, compararam-se os indicadores TLI e CFI relativo ao modelo a ser comprovado. De acordo com Damásio, (2013; cf. Moroco, 2010; Hair, Anderson, Tatham, \& Black, 2005) para que possa afirmar a invariância do modelo, espera-se que a diferença, específica do CFI e TLI, seja $\Delta<0,01$, entre ambos os estudos a fim de garantir a estrutura fatorial: sendo assim, dos 463 sujeitos da pesquisa, organizaram-se duas amostras, uma com 232 sujeitos (N1) e outra com $231(\mathrm{~N} 2)$. Desta maneira, $\mathrm{CFI}_{\text {Geral }}=0,99$ e $\mathrm{CFI}_{\mathrm{N} 1}=0,98$ e $\mathrm{CFI}_{\mathrm{N} 2}=0,99$ e para o TLI observou-se respectivamente, $\mathrm{TLI}_{\mathrm{Geral}}=0,99$ e $\mathrm{TLI}_{\mathrm{N} 1}=0,99$ e $\mathrm{TLI}_{\mathrm{N} 2}=0,98$. Com esses resultados, é possivel destacar que o modelo trifatorial da DASS-21 é invariate, isto é, independente da amostra, fatorialidade se mantém.

A partir desses resultados, tendo todos eles correspondidos ao que se pretendia, questionou-se quanto a possibilidade de uma avaliação a respeito dos fatores que, se pareados, quais apresentariam maiores escores entre si. Através de um teste t para amostra pareadas, observou-se, na Tabela 4, que o fator ansiedade apresentou maior escore quando pareado com o stress e depressão, tendo este último fator apresentado maior escore do que o fator do que o observado no stress. Ao salientar esse resultado é possível destacar uma hierarquia entre os escores dos fatores: Ansiedade > Depressão > Stress. É preciso também, chamar atenção para os efeitos correlacionais e entre esses fatores, os quais, não foram apenas significativos, mas, estiveram altamente correlacionados. 
Tabela 4: Escores médios do teste t pareado entre as variáveis do DASS-21.

\begin{tabular}{llcccc}
\hline Pares de variáveis & Média & d.p. & t & r \\
\hline \multirow{2}{*}{ Pares 1} & Stress & 8,62 & 8,91 & $-15,87^{*}$ & $0,89^{*}$ \\
& Ansiedade & 11,84 & 9,87 & & \\
& & & & & $0,85^{*}$ \\
\multirow{2}{*}{ Pares 2} & Stress & 8,62 & 8,91 & $-2,76^{*}$ & $0,86^{*}$ \\
& Depressão & 9,03 & 9,67 & & $-11,69 *$ \\
\multirow{2}{*}{ Pares 3 } & Ansiedade & 11,84 & 9,87 & & \\
& Depressão & 9,03 & 9,67 & & \\
\hline
\end{tabular}

Notas: d.p. $=$ desvio padrão; $\mathrm{r}=$ correlação de Pearson; * p-valor <0,05. Fonte: Autores.

Tendo garantido a estrutura fatorial da escala DASS-21 em uma amostra com trabalhadores distribuída em dois estados da região nordeste, pode-se afirmar que esta medida além de consistente, é convergente, a partir da avaliação dos indicadores psicométricos e a associação itens-fator, a proposta teórica abordada pelos autores (cf. Lovibond \& Lovibond, 1995; Machado \& Bandeira, 2013; Vignola \& Tucci, 2014; Patias, Machado, Bandeira, \& Dell'Aglio, 2016). Com a DASS-21 destinada à avaliação do transtorno emocional, procurou-se como dado adicional, avaliar a sensibilidade e a especificidade do instrumento na amostra em questão.

Embasado na orientação de um determinado tipo de cálculo com objetivo avaliar a quantificação e exatidão de um teste diagnóstico frente ao problema-fenômeno, a título de lembrança, a compreensão da ansiedade, depressão e estresse em trabalhadores, recorreu-se à análise de Curva ROC; esta, destina-se à avaliação das especificidades do instrumento de medida administrando numa pesquisa quantitativa com foco na avaliação comportamental ou atitudinal (Oliveira \& Andrade, 2002; Margotto, 2010). Para isso, calcularam-se as três dimensões do DASS-21 na amostra total, tendo com isso, observado na curva de ROC, indicadores estatísticos acima do eixo central (isto é, > 0,70), permitindo afirmar que tal medida apresenta uma sensibilidade diagnóstica relativo a proposta da DASS-21 (Figura 2).

Figura 2: Representação gráfica da especificidade e sensibilidade da DASS-21 em trabalhadores.

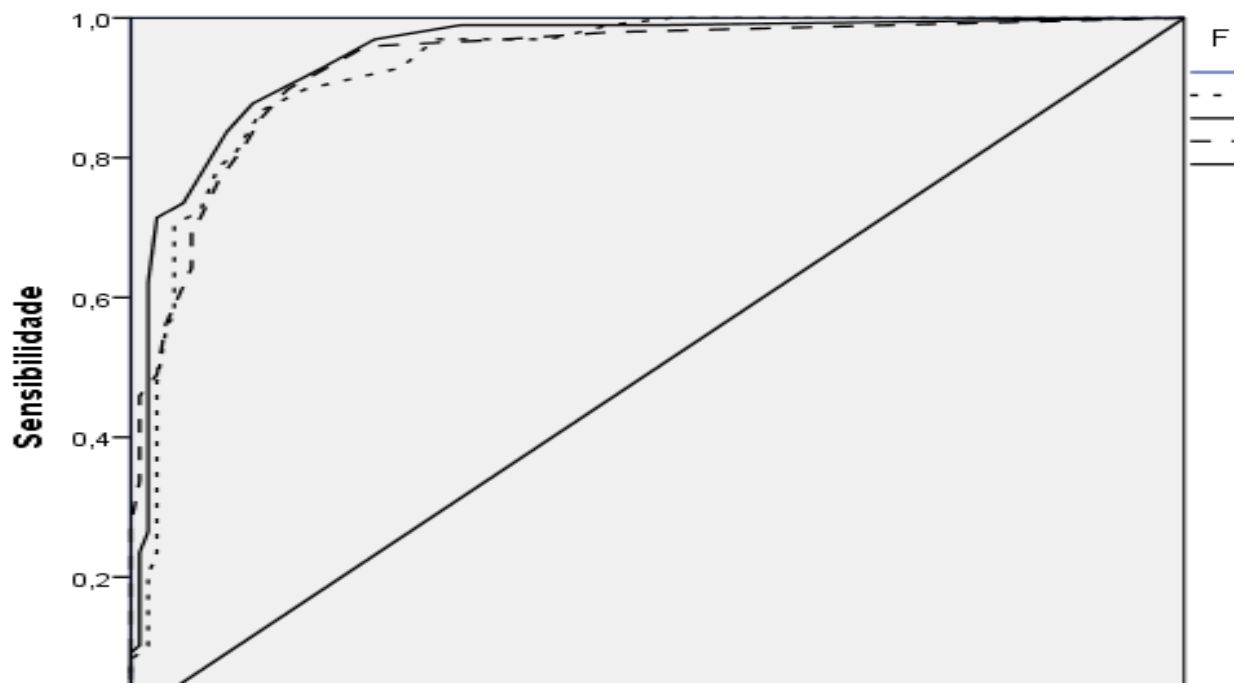

Fonte: Autores. 
$\mathrm{Na}$ Tabela 5, estão expressos os valores da área do ROC, os quais, variaram de 0,93 a 1,00, demonstrando que as probabilidades previstas são verdadeiras e correspondem bem ao efeito real esperado, isto é: a medida é sensível para a identificação do transtorno emocional em trabalhadores, podendo estabelecer com esta medida, a proposta de uma diagnose com base na perspectiva teórica e avaliativa em tais respondentes.

Tabela 5: Indicadores estatísticos da área de ROC para as dimensões da DASS-21

\begin{tabular}{cccccc}
\hline Construto & Área & erro & p-valor & $\begin{array}{c}\text { Limite } \\
\text { Inferior }\end{array}$ & Limite Superior \\
\hline DASS-21 & 1,00 & 1,00 & 0,001 & 0,99 & 1,00 \\
\hline Ansiedade & 0,93 & 0,17 & 0,001 & 0,90 & 0,97 \\
\hline Estresse & 0,95 & 0,15 & 0,001 & 0,92 & 0,98 \\
\hline Depressão & 0,93 & 0,16 & 0,001 & 0,90 & 0,97 \\
\hline
\end{tabular}

Fonte: Autores.

A partir desses resultados, os múltiplos indicadores estatísticos garantiram não apenas a consistência psicométrica da escala DASS-21 em trabalhadores, mas, também, a qualidade da medida na referida amostra. Trata-se de um instrumento que se revelou fidedigno e consistente, corroborando tanto a estrutura trifatorial quanto a capacidade desta medida para avaliar o problema do transtorno emocional, seja em sua consistência na relação medida-construto, seja quanto fator diagnóstico.

Efetuou-se também, uma Anova one-way, associada ao teste post-hoc de Scheffé, com o objetivo de avaliar as diferenças nas pontuações médias nas variáveis dependentes (DASS-21[pontuação total] e dos seus respectivos fatores, Ansiedade, Estresse e Depressão) versus independente (cidade [João pessoa-PB e Natal-RN] e tipo de empresa [pública e privada]). Considerando essa análise, observou-se, no que se refere ao tipo de empresa, a existência de resultados significativos em todas a dimensões do DASS-21, tendo uma influência do efeito direto nos trabalhadores da organização privada, a qual, apresentou escores médios superiores no estresse $\left(\mathrm{F}_{(1,461)}=122,55, p<0.001\right)$, na ansiedade $\left(\mathrm{F}_{(1,461)}=180,15, p<0.001\right)$ e na depressão $\left(\mathrm{F}_{(1,461)}=126,36, p<0.001\right)$.

No que diz respeito, ao contexto geográfico em que foi coletado a amostra, isto é, a cidade de João Pessoa-PB e de Natal-RN, observou-se que os trabalhadores da cidade de Natal-RN, os quais, apresentaram escores médios superiores, no estresse $\left(\mathrm{F}_{(1,461)}=142,28, p<0.001\right)$, na ansiedade $\left(\mathrm{F}_{(1,461)}=120,91, p<0.001\right)$ e na depressão $\left(\mathrm{F}_{(1,461)}=156,68, p<0.001\right)$.

\section{Discussão}

O estudo em questão pretendeu verificar a medida DASS-21 em uma amostra com distintos trabalhadores. Esta pesquisa, por sua vez, teve como orientação os variados estudos, os quais, supracitados, sobre esta escala em diferentes contextos amostrais de vários países. Estes, apresentavam algumas diferenças na sua organização fatorial, por exemplo: alguns estabeleciam um fator, outros dois e a maior parte três fatores.

Desta maneira, avaliou-se a partir dos critérios psicométricos considerado na proposta original apresentado por Lovibond e Lovibond (1995), referente ao modelo trifatorial. Com isso, comparando os modelos fatoriais, corroborou-se não apenas uma organização itens-fator com três fatores revelando indicadores psicométricos confiáveis, bem como, tratou-se de uma fatorial adequadamente parcimoniosa (ver tabela 1 e 2).

Os pressupostos empíricos, previamente considerados nas análises das tabelas supracitadas, permitiu que se avaliasse o quanto tal construto seria válido na sua estrutura empírica e teórica; sendo assim, foi verificado no cálculo do CC e VME esta condição, a qual, demostrou que o pretenso construto é confiável em sua estrutura trifatorial. Algo muito importante, é que 
estes fatores estão inter-relacionados, isto é: provavelmente, o respondente que apresentar escores mais alto em um dos fatores, também, pontuará alto nos demais. Tais resultados, são confirmados tanto por sua significância preditiva quanto pela baixa inflação da variância do fator.

No contexto da qualidade da medida, a realização do cálculo de invariância foi da estrutura fatorial garantia que a organização trifatorial é a mais adequada, pois, independente da amostra (mesmo com características sóciodemográficas semelhantes dos respondentes), tal modelo revelou-se consistente e numa estrutura contínua. Além de corroborar as propostas de pesquisadores brasileiros que propuseram uma condição fatorial (cf. Patias, Machado, Bandeira, \& Dell'Aglio, 2016; Martins Martins, Silva, Maroco, \& Campos, 2019) semelhante ao que se pretendeu verificar nesta pesquisa, no presente estudo, compararam-se uma diversidade de modelos estruturais, atitude essa, distinta dos estudos supracitados.

Com a qualidade a comprovada, observou-se a existência de uma possível hierarquia de contexto 'intra-influente' da medida. Avaliando o pareamento entre os fatores do DASS-21 (ver tabela 4), apesar de identificar que nestes pares exista sempre um dos fatores que se sobressai em pontuação média, na avaliação geral, destaca-se que, sempre, a ansiedade tem escore maior do que os outros fatores, seguido de uma segundo escore maior (neste caso, a depressão), finalizando com o estresse.

Tendo observado que este construto contempla também, uma área da saúde mental, muito específica, optou-se em avaliar de forma diagnóstica, o DASS-21 (ver Figura 1); tal fato motivou devido a condição de não ter encontrado, nos estudos supracitados, uma avaliação nesta direção. Sendo assim, todas as dimensões revelaram sensibilidade na avaliação desse construto nos trabalhadores.

Apesar de observar um resultado bastante promissor, sua saliência psicométrica não poderá ser indicada quanto exclusividade para um tratamento psicológico ou psiquiátrico, afinal, todos os respondentes não estão no contexto clínico, não houve uma detecção prévia referente a existência desses transtornos nos respondentes.

Todavia, considerar tais avaliações contribuiria para um processo mais pedagógico na avaliação desses fenômenos em trabalhadores, inibindo ou até desenvolver uma maior acurácia na verificação dos comportamentos daqueles trabalhadores que possam apresentar um traço do transtorno emocional.

Para isso, chama-se atenção para uma avaliação mais específica e contínua em trabalhadores de organização privada, já que tais resultados, na Anova, revelaram que os maiores escores na ansiedade, depressão e estresse, esteve para tais trabalhadores. Mas, um detalhe poderá ser destacado: por que os trabalhadores do Rio Grande do Norte tiveram maiores escores nestas variáveis?

Uma explicação deste fenômeno, poderia pautar-se na condição social, econômica e política no próprio Estado na época da coleta dos dados. Este, estava com o pagamento dos salários atrasados (e até o momento deste artigo, não foram atualizados os salários dos funcionários) afetando não apenas o contexto comercial em geral, mas, também, a estrutura e funcionalidade das organizações privadas, as quais, tiveram que se reorganizar em números e especificações de funcionários. Condição que permite refletir, que nos processos da identificação dos traços de transtorno emocional, o contexto social poderá ter uma grande contribuição na identificação e promoção de fatores de proteção.

\section{Conclusão}

A perspectiva do transtorno emocional em trabalhadores, refletido na escala DASS-21, apresentou adequada validade, confiabilidade e invariância fatorial entre os respondentes. Esta escala, caracteriza-se não apenas quanto a sua capacidade de avaliar três construtos psicológicos da saúde mental numa só medida, mas, também, por contribuir que a ansiedade, depressão e estresse não ocorre de forma isolada e sim, quanto sistemas interdependentes na avaliação destas variáveis.

Este artigo, teve o propósito de compreender a estruturação e diferenças dessas variáveis em diferentes profissionais. 
Com isso, pode-se garantir que o objetivo geral da pesquisa foi atendido, pois, foi possível identificar tanto a qualidade fatorial e sua confiabilidade, quanto a sua sensibilidade diagnóstica em trabalhadores.

Apesar desses resultados revelarem indicadores psicométricos aceitáveis, tendo alcançado os objetivos pretendidos, acredita-se que em estudos futuros poderia ser útil avaliar a relação de tais variáveis associadas a outras variáveis do comportamento organizacional (por exemplo, cultura organizacional, intenção a rotatividade, suporte organizacional, entre outras) em empresas públicas, privadas e mistas, bem como, considerar o tempo de serviço na avaliação dessas variáveis.

Um outro contexto que seria bastante importante é comparar aqueles respondentes com e sem diagnóstico do transtorno emocional; e, também, verificar o quanto essas variáveis se diferenciam em trabalhadores no âmbito internacional, objetivando avaliar os diferentes contextos políticos, sociais e econômicos em relação as políticas implementadas no espaço laboral.

\section{Referências}

Abella, L. E. E \& Zapata, D. I. (2011). Relación entre capital psicológico y la conducta de compartir conocimiento en el contexto del aprendizaje organizaciona. Ata Colombiana de Psicología, 14(1), 61-70.

Adam, F., \& Rončević, B. (2003). Social Capital: Recent Debates and Research Trends. Social Science Information, $42(2), 155-183$.

American Psychiatric Association - DSM-5 (2014). Manual Diagnóstico e Estatístico de Transtornos Mentais (DSM-5). Artmed.

Antunes, A, Caetano, A. \& Cunha, M. P. (2013). O papel do capital psicológico na criação de valor para as organizações. Revista Portuguesa e Brasileira de Gestão. 12 (3), 2-10.

Avey, J. B., Luthans, F., \& Jensen, S. (2009). Psychological capital: A positive resource for combating stress and turnover. Human Resource Management, 48(1), 677-693.

Avey, J. B., Luthans, F., Smith, R. M., \& Palmer, N. F. (2010). Impact of positive psychological capital on employee well-being over time. Journal of Occupational Health Psychology, 15(1), 17-28.

Avey, J.B.; Reichard, R. J. Luthans, E.; \& Mhatre, K. E. (2011). Meta-Analysis of the Impact of Positive Psychological Capital on Employee Attitudes, Behaviors, and Performance. Human Resource Development Quarterly, 22(2), 127-152.

Bandura, A. (1977). Self-efficacy: Toward a unifying theory of behavioral change. Psychological Review, 84(2), 191-215.

Baptista, M. N., \& Carneiro, A. M. (2011). Validade da escala de depressão: relação com ansiedade e stress laboral. Estudos de Psicologia (Campinas), 28(3), 345-352. https://dx.doi.org/10.1590/S0103-166X2011000300006

Barreto, I. S., \& Teodoro, M. L. M. (2018). Problemas emocionais e de comportamento, vulnerabilidade cognitiva e estresse: uma revisão narrativa. Contextos Clínicos, 11(1), 37-58. https://dx.doi.org/10.4013/ctc.2018.111.04

Borim, F. S. A., Barros, M. B. de A., \& Botega, N. J. (2013). Transtorno mental comum na população idosa: pesquisa de base populacional no Município de Campinas, Brasil. Cadernos de Saúde Pública, 29(7), 1415-1426. https://dx.doi.org/10.1590/S0102-311X2013000700015

Brixner, J., Formiga, N. S., \& Navarro, M. M. (2019). Professor, você se sente bem?! os fatores de proteção do distúrbio psíquico em professores de escola pública de Brasília, Distrito Federal, Brasil. Riga Latvia, European Union: Nova Edições Acadêmicas.

Brown, T., Chorpita, B., \& Barlow, D. (1998). Structural relationships among dimensions of the DSM-IV anxiety and mood disorders and dimensions of negative affect, positive affect, and autonomic arousal. Journal of Abnormal Psychology, 107(2), 179-192.

Clapp-Smith, R.; Vogelgesang, G. \& Avey, J. (2009) Authentic Leadership and Positive Psychological Capital: The Mediating Role of Trust at the Group Level of Analysis. Organizational Studies, 15(3), 227-240,

Clark, L., \& Watson, D. (1991). Tripartide model of anxiety and depression: Psychometric evidence and taxonomic implications. Journal of Abnormal Psychology, 100(3), 316-336.

Coleman, J. S. (1988). Social Capital in the Creation of Human Capital. The American Journal of Sociology, 94(1), 95-120.

Cunha, M.; Rego, A.; Lopes, M. \& Ceitil, M. (2008). Organizações positivas: manual de trabalho e formação para desenvolver as forças dos indivíduos e dasorganizações. Sílabo.

Dyer, L., \& Reeves, T. (1995) Human resources strategies and firm performance: what do we know and where do we need to go? International Journal of Human Resource Management, 6(3), 656-670.

Gazalle, F. K., Lima, M. S., Tavares, B. F., \& Hallal, P. C. (2004). Sintomas depressivos e fatores associados em população idosa no Sul do Brasil. Revista de Saúde Pública, 38(3), 365-371. https://doi.org/10.1590/S0034-89102004000300005 
Gelder, M., Gath, D., Mayou, R., \& Cowen, P. (1996). Oxford texbook of psychiatry (3rd ed.). Oxford: Oxford University Press.Hackman, J. R. (2009). The perils of positivity. Journal of Organizational Behavior, 30(1), 309-319.

Gratton. L., \& Ghoshal, S. (2003). Managing personal human capital: New ethos for the 'volunteer’ employee. European Management Journal, 21(1), 1-10.

Hamaideh, S. H. (2017). Alexithymia among Jordanian university students: Its prevalence and correlates with depression, anxiety, stress and demographics. Perspective Psychiatrique Care, 0(0), 1-7.

Kogut, B., \& Zander, U. (1996). Knowledge, Market failure and multinational enterprise: a reply. Organization Science, 7(1), 502-518.

Larson, M.; Luthans, F. (2006) Potential added value psychological capital in predicting work attitudes. Journal of Leadershi and Organizational Studies, Thousand Oaks, 13(1), 45-61.

Le, K. B. et al (2006). Minimal criteria for defining multipotent mesenchymal stromal cells. The International Society for Cellular Therapy position statement. Journal Cytotherapy, 8(4), 22-36.

Leite, M. P. \& Souza, A. N. (2006). Condições do trabalho e suas repercussões na saúde dos professores da educação básica no Brasil. Unicamp/Funcamp. Campinas: SP.

Lopez, S. J., \& Snyder, C. R. (Eds.). (2009). Handbook of positive psychology (2nd ed.). Oxford University Press.

Lovibond, P., \& Lovibond, S. (1995). The structure of negative emotional states: Comparison of the depression anxiety stress scales (DASS) with the Beck Depression and Anxiety Inventories. Behaviour Research and Therapy, 33(3), 335-343.

Luthans, F. (2002). The need for and meaning of positive organizational behavior. Journal of Organizational Behavior, 23(6), 695-706.

Luthans, F., \& Avolio, B. J. (2014). Brief Summary of Psychological Capital and Introduction to the special Issue. Journal of Leadership \&Organizational Studies, 21(2), 125-129.

Luthans, F., \& Youssef, C. M. (2004). Human, social, and now positive psychological capital management: Investing in people for competitive advantage. Journal Organizational Dynamics, 33(2), 143-160.

Luthans, F., Norman, S. M., Avolio, B. J., \& Avey, J. B. (2008). The mediating role of psychological capital in the supportive organizational climate-employee performance relationship. Journal

Luthans, F., Youssef, C. \& Avolio, B. (2007). Psychological Capital. Developing the Human Competitive Edge. Oxford University Press.

Margotto, P. R. (2010). Curva ROC como fazer e interpretar no SPSS. Disponível em: http://www.paulomargotto.com.br/documentos/Curva_ROC_SPSS.pdf

Margotto, P. R. (2010). Curva ROC como fazer e interpretar no SPSS. Disponível em: http://www.paulomargotto.com.br/documentos/Curva_ROC_SPSS.pdf

Martins, B. G., Silva, W. R., Maroco, J., \& Campos, J. A. D. B. (2019). Depression, Anxiety, and Stress Scale: psychometric properties and affectivity prevalence. Jornal Brasileiro de Psiquiatria, 68(1), 32-41. https://dx.doi.org/10.1590/0047-2085000000222

Martins, B. G., Silva, W. R., Maroco, J., \& Campos, J. A. D. B. (2019). Escala de Depressão, Ansiedade e Estresse: propriedades psicométricas e prevalência das afetividades. Jornal Brasileiro de Psiquiatria, 68(1), 32-41. https://dx.doi.org/10.1590/0047-2085000000222

Masten, C. L. et al. (2010). Witnessing peer rejection during early adolescence: Neural correlates of empathy for experiences of social exclusion. Soc Neurosci, 5(5-6), 496-507, out.

McCauley, E., Katon, W., Russo, J., Richardson, L., \& Lozano, P. (2007). Impact of anxiety and depression on functional impairment in adolescents with asthma. General Hospital Psychiatry, 29(3), 214-222. https://doi.org/10.1016/j.genhosppsych.2007.02.003

Melo, A. K., Siebra, A. J., \& Moreira, V. (2017). Depressão em Adolescentes: Revisão da Literatura e o Lugar da Pesquisa Fenomenológica. Psicologia: Ciência e Profissão, 37(1), 18-34. https://dx.doi.org/10.1590/1982-37030001712014

Morin, P. V. (2016). Transtornos mentais comuns em trabalhadores rurais que utilizam agrotóxicos. Dissertação (mestrado) Universidade Regional do Noroeste do Estado do Rio Grande do Sul (Campus Ijuí).

Nahapiet, J., \& Ghoshal, S. (1998) Social capital, intellectual capital, and the organizational advantage. Academy of Management, 23(2), 1-25, abril.

Neveu, E. Introdução aos Estudos Culturais. Tradução de Marcos Marcionilo. Parábola Editorial, 2004

Nguyen, T. (2012). Transitivity Analysis of Heroic Mother by Hoa Pham. International Journal of English Linguistics, 2 (4), 85-100.

Norman, S. M., Avey, J. B., Nimnicht, J. L., \& Graber-Pigeon, N. P. (2010). The interactive effects of psychological capital and organizational identity on employee citizenship and deviance behaviors. Journal of Leadership and Organization Studies, 17(1), 380-391.

Oliveira, J. G. C., \& Andrade, F. W. M. (2002). Comparação entre medidas de performance de modelos de credit scoring. Tecnologia de Crédito, 33 , 35-47.

Pai, D. D., Lautert, L., Souza, S. B. C., Marziale, M. H. P., \& Tavares, J. P. (2015). Violência, burnout e transtornos psíquicos menores no trabalho hospitalar. Revista da Escola de Enfermagem da USP, 49(3), 457-464. https://dx.doi.org/10.1590/S0080-623420150000300014

Pais-Ribeiro, J. L., Honrado, A., \& Leal, I. (2004). Contribuição para o estudo da Adaptação Portuguesa das Escalas de Ansiedade, Depressão e Stress (EADS) de 21 Itens de Lovibond \& Lovibond, Psicologia, Saúde \& Doenças, 1(1), 229-239. 
Research, Society and Development, v. 10, n. 7, e26910715572, 2021

(CC BY 4.0) | ISSN 2525-3409 | DOI: http://dx.doi.org/10.33448/rsd-v10i7.15572

Patias, N. D., Machado, W. L., Bandeira, D. R., \& Dell'Aglio, D. D. (2016). Depression Anxiety and Stress Scale (DASS-21) - Short Form: Adaptação e Validação para Adolescentes Brasileiros. Psico-USF, 21(3), 459-469. https://dx.doi.org/10.1590/1413-82712016210302

Seligman, M. E. (2005). Positive Psychology, Positive Prevention, and Positive Therapy. In S. J. Lopez \& C. R. Snyder (Eds.). Handbook of positive psychology. Oxford University Press.

Snyder, C. R., \& McCullough, M. E. (2000). A Positive Psychology Field of Dreams: "If You Build it, They Will Come..."Journal of Social and Clinical Psychology, 119(1), 151-160.

Terra, F. S. (2010). Avaliação da ansiedade, depressão e autoestima em docentes de Enfermagem de universidades pública e privada. Tese de doutorado (USRibeirão Preto). file:///C:/Users/nsfor/Downloads/Fabiosouzaterra.pdf

Watson, D., Clark, L., Weber, K., Assenheimer, J., Staruss, M., \& McCormick, R. (1995a). Testing a tripartide model: II. Exploring the symptom structure of anxiety and depression in student, adult, and patient samples. Journal of Abnormal Psychology, 104(1), 15-25.

Watson, D., Weber, K., Assenheimer, J., Clark, L., Staruss, M., \& McCormick, R. (1995b). Testing a tripartide model: I. Evaluating the convergenct and discriminant validity of anxiety and depression symptom scales. Journal of Abnormal Psychology, 104(1), 3-14.

Youssef, C.; Luthans, F. (2011) Positive psychological capital in the workplace: where we are and where we need to go. In: Sheldon, M.; Kashdan, T.; Steger. (org.). Designing the future of positive psychology. (p. 335-364) Oxford. Unive 